Review Article

\title{
Development of resistance to the effect of local anesthetic agents administered via various routes due to single or multiple, previous scorpion bites: a proposed hypothesis and reporting a yet unrecognized phenomenon
}

\begin{abstract}
"Failure" to achieve the local anesthetic block, is demoralizing, distressing and mystifying, especially, if the technique is standard, the knowledge/skill of the operator is above average and the concentration, volume and dose of the drug is correct. In spite of fulfilling all these pre-requisits, when there is non-adequate block or occurrence of partial and patchy block, the operator, becomes a subject of ridicule and mockery. In the literature, there are multiple reports of 'resistance to local anesthetics', which till now have been relegated to the realm of cynicism!
\end{abstract}

During routine practice, we encountered persistently, a spate of 'failed local anesthetic blocks', via, various routes, like sub-arachnoid, supra-clavicular/axillary brachial plexus and peribulbar. This was happening in spite of everything being done correctly and even in expert hands! After ruling out all probable causes and on detailed enquiry, the only common denominator which emerged was, "previous history of single/multiple scorpion bites", as most of our rural patient population belonged to the area "endemically infested by scorpions". During the course of their routine agricultural work, it was of common occurrence, to be bitten by the scorpions at least once but in many, multiple times, in their life time. In these "Failure to achieve a block" patients, it was established that they had concurrent development of 'failure to produce numbness' even with local anesthetic infiltration/injection in the distribution of superficial/regional nerves.

Encouraged, we conducted a case control study. The patients for, elective/planned surgical procedures to be carried out with sub-arachnoid block were randomly selected and delegated to either of the two groups, patients giving history of one/multiple scorpion bite/s and with history of 'no bites'. Rest of the management was similar in both the groups. In the bites group, there were 'complete failure or patchy block/delayed onsets', while in the 'nonbites' all successful blocks. This confirmed that, there appears to be a direct correlation between the previous scorpion bite/s and development of "resistance" to the effect of Local anesthetics, an apparent cause yet unreported.

Implications are scorpions exist almost all over the world. Persons after scorpion-bite, recover and lead normal life. These persons, when exposed to local anesthetics may exhibit 'mysterious/unexplainable resistance' to the effect of local anesthetics. This causes distress for operator, patient, wasted time/effort, disrepute of local anesthetics/techniques and discouragement for the young physicians. This yet unreported phenomenon, when recognized and history of scorpion-bite elicited, the choice of anesthesia can be made accordingly.

So we propose that, previous single/multiple scorpion bite/s may lead to development of resistance to Local Anesthetic Agents!

Keywords: previous scorpion bite, single or multiple, development of 'resistance'; effect of local anesthetic agents, hypothesis
Volume 3 Issue 5 - 2015

Mridul M Panditrao,' Minnu M Panditrao,' Aditi M Panditrao ${ }^{2}$

'Department of Anesthesiology and Intensive Care, Adesh University, India

${ }^{2}$ Department of Pharmacology, Bharti Vidyapeeth Medical College, India

Correspondence: Mridul M Panditrao, Department of Anesthesiology and Intensive Care,Adesh University, India, Email drmmpraol@gmail.com

Received: October 28, 2015 | Published: November 27, 2015

\section{Introduction}

It is a common clinical practice to administer local anesthetic agents via various routes to achieve 'Optimum Local Anesthetic Blocks,' as they are conventionally referred to. The main aim is to produce adequate analgesia for various indications, like surgical procedures or as a pre-requisite to interventional procedures or as a part of pain management strategy. It may be conducted by any and all the clinicians, but most commonly performed by anesthesiologists, as a modality of their routine anesthetic practice. It is very disheartening for the operator, when despite using correct amount of drug, visà-vis, dose $(\mathrm{mg} / \mathrm{kg})$, concentration and volume and technically correct method of injection of the correct drug at the correct place does not produce the anticipated results and he/she has to face the skepticism from the audience in the operating theater, not to mention, start preparing for the suitable alternative/back up plan, like supplementation with sedation or conversion to general anesthesia. 
There are reports of "not able to achieve" the adequate sensory/ motor effect when local anesthetic is administered either via neuraxial route, for peripheral/ regional nerve blocks or as local infiltration, where, because of lack of any substantial and sustainable explanation, the inability to achieve the block had to be attributed to the so-called "resistance to, or failure of effect of local anesthetics". ${ }^{1-3}$ Resistance to the effect of local anesthetic agents is an uncommon but not an unknown phenomenon. The multifactorial etiology has been well documented. ${ }^{4-18}$ In spite of many of these available case reports and series, the ambiguity persists and authenticity of this phenomenon is still not very well established.

\section{Background information}

It all started, when in a 65 years old woman, posted for total vaginal hysterectomy, we encountered a complete failure of the intra-thecal analgesia, in spite of two successive administrations of Bupivacaine heavy $0.5 \%$ via subarachnoid route, one of them administered by a clinician of the stature of professor and clinical experience/expertise of more than 25 years. So General anesthesia had to be employed as a suitable alternative, with unremarkable and uneventful intraoperative and post-operative course. In the post-operative period, after proper explanation of 'possible unusual condition i.e. resistance to local anesthetics' and after obtaining consent, Inj. Bupivacaine, was infiltrated in the distribution of the all the three nerves of the left hand and forearm (Median, Ulnar and Anterior Interosseous), what is referred to as "Wrist Block". The complete failure of the effect of local anesthetic to produce any significant 'sensory' block in the overlying skin in the area of hand/fingers, as well as 'motor' block in the muscles of fingers (interossei and lumbricals), raised possibility of an unusual but plausible hypothesis of "resistance to local anesthetics". There was no relevant history, which could attribute this to genetic/ heredo-familial etio-pathogenesis. The only emergent relevant history was of scorpion bites, twice in past, first one at the age of 17 years and second about 8 months back, at multiple sites. ${ }^{2}$

In addition, in another 80 years old man posted for cataract surgery under peri-bulbar block, the block was administered by a senior ophthalmologist, again with clinical experience/expertise of almost 25 years, with $2 \%$ lidocaine and to everybody's utter surprise; there was absolutely no effect, so she supplemented it with further injections. Even after doing so, only partial and patchy/ineffective block was achieved and surgery had to be completed under supplemental intravenous sedation and opioid analgesia..$^{3-18}$ In the post-operative period, patient gave past history of scorpion bite three times, at the age of 27 years on his right foot, another 8-9 years back and last, 6-7 months back on his right hand. After obtaining necessary consent and administering the local infiltration in the ventral aspect of forearm, with $2 \%$ lidocaine, again when failed to produce the anticipated block (just like first patient), we were determined to test this hypothesis.

So with the awareness about such cases spreading, it became a routine to elicit history of scorpion bite as a part of routine and essential measure, while performing evaluation in the Anesthesia preoperative clinic as well as in the pre-anesthetic ward rounds.

\section{Hypothesis}

\section{Our hypothesis is}

i. "Previous single or multiple scorpion bites lead to development of resistance to the effect of local Anesthetic drugs."

ii. A person, during his/ her life time, may have been bitten by a scorpion, be it once or multiple times, has completely recovered from effects of the scorpion venom and is leading his/her normal life. In such a patient when posted for any surgery, where the Local Anesthetic drugs are used to achieve analgesia, there is varying degree of failure of the effect of the local anesthetic drug, ranging from complete failure to delayed onset and incomplete/partial/ patchy block. The route of administration of the local anesthetic has no significant role, but the number of bites, duration between the last bite and the exposure to the local anesthetic have definite role to play; more the number of bites and lesser the duration between last bite and the exposure to the local anesthetic, more the chances of complete failure of the effect of local anesthetics.

iii. The scorpion venom and the local anesthetic drugs have a common site for binding, the 6th segment of domain four of the alpha subunit (IV-S6). ${ }^{19}$ The scorpion venom is a mixture of multiple peptides with potent antigenicity. We hypothesize that, exposure to the antigens in scorpion venom, due to previous scorpion bite leads to formation of antibodies against them, which remain in the systemic circulation, throughout their life. Once these patients are exposed to the local anesthetic agents in future, there is a competitive antagonism, at the common binding site (IV-S6) leading to failure/ ineffectiveness/ partial effectiveness of the local anesthetics.

iv. More the number of the bites/more recent the last bite and lesser the duration between the bite and the time of the exposure to local anesthetics, more pronounced is the failure/ ineffectiveness of the local anesthetics.

\section{Evaluation of Hypothesis/ Idea}

\section{Materials and methods}

As a logical next step, a randomized, case control study was designed, wherein 70 randomly selected patients posted for routine elective surgical procedures under proposed spinal (sub-arachnoid) analgesia were divided into two groups of 35 patients each, with patients giving history of scorpion bite (single/multiple) in one group while, patients with no bite in second group.

Rest of the protocol for management was absolutely common for all the patients in both the groups of patients. It included:

a. Pre-operative preparation, investigations, assessment, medications

b. Wheeling in the OR, at the time of surgery, connecting to monitors

c. Multi-parametric monitoring: Pulse/ ECG, Pulse oxymetry ( oxygen saturation), Non-invasive blood pressure(NIBP)

d. Initiation of intra venous infusion with help of $20 \mathrm{gz}$ IV cannula and an infusion of ringer lactate

e. Achievement of sub-arachnoid block with $27 \mathrm{gz}$. Quincke type of spinal needle in L2-L3 or L3- L4 interspaces, with, $3.5 \mathrm{ml}$ (17.5mg) of $0.5 \%$ Bupivacaine heavy ( $17.5 \mathrm{mg}$ ) after free flow of CSF. This time was considered as T0

f. The assessment of the sensory block with pinprick method with help of blunted 24 gauze hypodermic needle and for assessment of motor block Bromage scale was applied.

The anesthesiologist assigned to do the assessment of the effect of block was same for all cases and controls and had been blinded to the history of scorpion bite. 
According to pre-decided end points, times of onsets of blocks, both sensory and motor (T1 and T2), peak of the action of blocks, both sensory and motor ( $\mathrm{T} 3$ and $\mathrm{T} 4)$.

The block was considered to be adequate when level of sensory/ motor block of up to T6- T8 was achieved. Ifin spite of waiting for nearly 20 minutes neither adequate sensory nor motor (or both) blocks were achieved, then the technique of anesthesia was converted to standard balanced general anesthesia (GA) using pre oxygenation, induction with protocol, intubation after paralyzing dose of rocuronium and maintain on gas mixture, rocuronium \&controlled ventilation.

\section{Results}

Total number of patients studied were 70 , equally distributed in the two groups of 35 each. There was no statistical difference between the both the groups, when compared for age, gender, American society of anesthesiologists (ASA) physical status, experience of anesthesiologists performing the blocks, types of surgical procedures being performed and the site of spinal block.

In the 'Bite' group $(\mathrm{n}=35), 23$ patients $(65.7 \%)$ had single bite, 4 $(11.4 \%)$ had two, $3(8.6 \%)$ had 3 and $5(14.3 \%)$ had 4 or more bites. All of these patients exhibited longer time for the onset of the sensory (T1 :2.31 \pm 1.68 minutes as compared to $1.63 \pm 0.84$ minutes in "Nonbite" group, $\mathrm{p}$ of 0.0340$)$ and motor block (T2 : 2.91 \pm 1.80 minutes as compared to $1.80 \pm 0.83$ minutes in non-bite group, $\mathrm{p}$ of 0.001$)$ and longer time to reach the peak of both sensory (T3:6.45 \pm 2.83 minutes as compared to3.66 \pm 0.94 minutes of non-bite group, p of 0.000 , highly significant) and motor block (T4: $7.83 \pm 3.95$ minutes as compared to $4.46 \pm 1.40$ minutes of non-bite group, $\mathrm{p}$ of 0.000 , highly significant).

In this same group, four patients exhibited incomplete sensory block, five patients had incomplete motor block and in two patients both modalities of spinal block failed. All these patients received general anesthesia.

\section{Discussion}

We decided to use sub-arachnoid block as our trial model, because, it is the most commonly performed modality of local anesthesia, it is comparatively easier to perform, economical and when performed even by the clinicians with lesser experience, the chances of 'failure' are practically minimal.

Remarkable finding that, all the patients in the 'Bite' group had either complete failures, partial/patchy blocks, delayed onsets of both sensory/ motor blocks, almost all had delayed onsets of action for sensory/motor blockade and not a very smooth and easy intraoperative course ( 4 patients had no sensory block, 5 patients had no motor block while, 2 patients had failure of both sensory/ motor blocks and had to be given the general anesthesia), while all the patients had adequate block in 'Non-bite' group, suggests that, there was some phenomenon, responsible for these differences, which clinically as well as statistically were significant. ${ }^{20}$

Logically a tentative inference can be drawn from all these findings that, there appears to be a tangible association between the single/ multiple scorpion bites and development of resistance to the "effect of local anesthetics'. The more the number of bites or more recent the bites, more the extent of failure of the block. The patients with maximum number of bites (3-4) and that also in the most recent past, had almost complete failure of the block.
Local anesthetics act by binding with $\mathrm{Na}+$ channels in the closed, open, and inactivated states. Specifically, structurally the sodium channel has three sub- units e.g. alpha, beta- 1 and beta- 2 subunits. The alpha subunit has further four homologous domains (I-IV) and each of these domains is made up of six trans-membrane segments (S1-S6). Mechanism of the action of the Local anesthetic drugs is believed to be due to an interaction with the 6th segment of domain four of the alpha subunit (IV-S6). ${ }^{19}$ Resistance to local anesthetics may be related to the alpha subunit of the sodium channel and more specifically to the 6th segment of domain four of this subunit.1

Hottentotta tamulus, the Indian red scorpion, is a species of scorpion, belonging to the family Buthidae. It occurs in most of India, ${ }^{21}$ and is the same species commonly found in the geographical location where our patients come from. Almost all the varieties occurring in the tropical and subtropical regions including some of the islands, in the world, belong to the same family, Buthidae. ${ }^{22}$

Scorpion venom of this family contains multiple toxins, biogenic amines, enzymes, salts, unidentified substances and water. These toxins, (which are low molecular weight, thermo-stable, basic proteins), are known to affect sodium ion channels, potassium ion channels and calcium ion channels. In fact the severity/toxicity of these substances has been attributed to this effect. ${ }^{22,23}$ The binding site of these at the sodium channels is the same as that of the local anesthetics, viz; 6th segment of domain four of the alpha subunit (IV-S6). ${ }^{19}$

\section{This has led us to hypothesize}

The antigenicity of the scorpion venom elicits a strong immunological response. This leads to formation of the antibodies against the binding site, the voltage gated sodium channels, more precisely, the 6th segment of domain four of the alpha subunit (IVS6). As the binding site/ receptor for the local anesthetics is same, when later on in life these sensitized patients are exposed to local anesthetics, these drugs are unable to bind to the common receptor (IVS6), because of competitive antagonism produced by these circulating antibodies. More the number of bites or more the recent are the bites, more potent the antagonism and thus more chances of "failure/ resistance to the effect of Local anesthetics". Nobody even bothers to elicit this previous history of scorpion bite, as the patients are otherwise not having any residual effect of that scorpion bite. In addition they have come for totally unrelated condition requiring surgery which was planned to be done under some local anesthetic block. As now can be predicted, the result of this ill-fated block is, either complete or partial failure to the dismay of everybody concerned, more so the operator. It is practically impossible to pinpoint the plausible cause of this failure, often considered to be the technical/operator failure.

As the scorpions are distributed almost all over the world, this phenomenon has significance even from global point of view. Recently on the lines of our findings and supporting our hypothesis, more evidence has emerged, where, Issin $\mathrm{A}^{23}$ has reported a case of "Local anesthetic resistance in a young woman with history of scorpion bite". ${ }^{24}$ We are of the opinion as more and more researchers and clinicians become sensitized and aware, about our hypothesis, they will be able to correlate, it with their own experiences and report this elusive phenomenon.

There can be multiple factors which can influence and limit the study, most important being our inability, at present to demonstrate the presence of these antibodies, by doing appropriate immunological tests, as none exist at present. In addition there can be hypothetically, 
some genetic factors which might influence the action of local anesthetics, classical example being, genetic factors like being a Redhead, i.e. carrying a variants of the melanocortin-1 receptor $(M C 1 R)$ gene, can lead to resistance to not only local anaesthetics like novocaine, lidocaine but may make them resistant to effects of inhalational anaesthetic agents like desflurane. ${ }^{6,78}$ But our hypothesis comes nearer to conviction when, by the process of exclusion and circumspection, we reach the only possible inference, of correlation between these two phenomena.

\section{Conclusion}

We are proposing a hypothesis, that, the previous single or multiple scorpion bites can cause development of resistance to the effect of local anesthetic agents, administered via various routes. The more the number of bites and more recent the bite, more the chances of failure, patchy/incomplete the block, delayed onset of sensory and/or motor blockade, thus requiring either replacement with balanced general anesthesia or supplementation with some adjuvant like sedation. We firmly believe, it has immunological basis and the mechanism of this 'resistance' involves possible 'competitive antagonism' at the receptor site, the 6th segment of domain IV of alpha subunit of the sodium channels in the peripheral nerves, the common binding site for both the scorpion venom and the local anesthetics agents(IV-S6). ${ }^{1,24,25}$

\section{Consequences of the Hypothesis}

i. Scorpions are commonly found in the tropical/subtropical countries/geographical locations. In fact majority of the population of the world is residing in these areas logically scorpion bite is relatively a common, but not a life threatening phenomenon occurring in these areas.

ii. A person, bitten by the scorpion, goes through a short period of mild/ moderate pain/ tenderness at the site of bite, recovers and then forgets anything/everything about that, leading a normal life.

iii. This patient, in future, if exposed to the local anesthetics for any reason, does not remember anything about the 'previous scorpion bite/s' and elicits unusual 'resistance' to the effect of local anesthetics, causing complete/partial failure or ineffective/ inadequate/incomplete block, necessitating employment of an additional suitable/alternative and unplanned/emergency modality of anesthesia.

iv. All this leads to distress to the patient and the operator, not to mention wasted time, efforts and resources. In addition, causing the demoralizing effect of whole of this occurrence on the psyche of the operators, irrespective of their age/experience and skill.

v. When this happens repeatedly (unexplainable failure of the local anesthetic block, in spite of doing everything correctly), because of the fear of becoming the focus of ridicule/sarcasm, there develops great reluctance/fear on the part of the operators to perform the local anesthetic blocks/ techniques. Thus these very useful techniques fall in disrepute/disuse, therefore depriving the deserving patients, the benefits of these blocks.

vi. Ifthisis ( as we envisage is), one of the leading causes of development of resistance' to the effect of local anesthetics, as is our endeavor, if more and more clinicians and other health care personnel become acquainted with this phenomenon, in the pre-anesthetic/operative assessment, asking for/eliciting 'previous/past history of scorpion bite' will be incorporated, as a pre-requisite, especially in the above-mentioned geographical areas, endemic for the preponderance of scorpions( Tropical/ sub tropical regions, rural/ agricultural populations/ persons residing in mountainous/ rocky terrain).

vii. In the patients giving such a history, where ever possible the employment of Local anesthetics can be avoided and choice would be either the complete general anesthesia or one of its modifications (Conscious sedation, monitored anesthesia careMAC or GA).

viii. We feel this phenomenon also puts the actual concept of "Resistance to Local Anesthetic agents' as such, in the limelight and out of the realm of the controversy/ ambiguity. It significantly adds to the 'existing body of knowledge' on the topic.

ix. At present the only and relevant lacuna, which we feel and accept is our inability to confirm the existence of the 'antibodies' formed against the constituent proteins (antigens) in the scorpion venom, which produce competitive antagonism with the local anesthetics at the common binding site; the (IV-S6).

$\mathrm{x}$. Once this phenomenon is widely publicized and wider interest is generated, the relevant researchers will be able to confirm the existence and elaborate on the methods of quantifying of these antibodies.

xi. The general physicians/ other health care personnel, can actually educate the colleagues, patients and their relatives, especially from the geographical areas with preponderance of scorpions, to be more aware and volunteer with the history of previous scorpion bites, when getting admitted for surgical procedures.

\section{Conflicts of Interest statement}

\section{Source of Funding}

The authors declare that there was No source of any funding involved in the preparation of this manuscript. The authors confirm that there are no known conflicts of interest associated with this publication and there has been no significant financial support for this work that could have influenced its out-come.

\section{Authors Contribution}

The corresponding author conceptualized the main theme of the article. Remaining authors did most of the literature search, correlating the findings from literature with the proposed hypothesis. The corresponding author did most of the writing, while remaining two authors did most of the editing, revising and final corrections in the preparation of manuscript. In fact all the authors contributed almost equally.

\section{Declarations}

The authors want to confirm that all the authors had full access to all the data in the study and corresponding author takes the full and final responsibility for the decision to submit for publication.

\section{References}

1. Batas D, Nejad MRG Prabhu PK. Resistance to local anaesthetics: A case report. British Journal of Anaesthesia. 2011.

2. Panditrao MM, Panditrao MM, Khan MI, et al. Does scorpion bite lead to development of resistance to the effect of local anaesthetics? Indian J Anaesth. 2012;56(6):575-578.

3. Panditrao MM, Panditrao MM, Sunilkumar V, et al. Can repeated scorpion bite lead to development of resistance to the effect of local anesthetics? Maybe it does! Case Reports in Clinical Medicine. 2013;2:179-182. 
4. Noyan AMA, Mirghasemi AA, Peiravy SH. True resistance to local anesthetics, a case report. Tehran Univ Med J. 2007;64: 97-100.

5. Raw RM, Nwaneri ER. Spinal anesthetic block failure due to the hyperbaric nature of the chloroprocaine local anesthetic. Ambul Surg. 2010;16 99-102.

6. Binkley CJ, Beacham A, Neace W, et al. Genetic Variations associated With Red Hair Color and Fear of Dental Pain, Anxiety Regarding Dental Care and Avoidance of Dental Care. JADA Continuing Education. J Am Dent Assoc. 2009;140(7): 896-905.

7. Liem EB, Joiner TV, Tsueda $\mathrm{K}$, et al. Increased sensitivity to thermal pain and reduced subcutaneous lidocaine efficacy in redheads. Anesthesiology. 2005;102(3):509-514.

8. Liem EB, Lin CM, Suleman MI, et al. Anesthetic requirement is increased in redheads. Anesthesiology. 2004;101(2):279-283.

9. Shrestha AB, Shrestha CK, Sharma KR, et al. Failure of subarachnoid block in Cesarean section. Nepal Med Coll J. 2009;11(1):50-51.

10. Harrisson DA, Langham BT. Spinal anaesthesia for urological surgery. A survey of failure rate, post dural puncture headache and patient satisfaction. Anaesthesia. 1992;47(10):902-903.

11. Guinard JP, Carpenter RL, Smith HS. A prospective evaluation of the failure rate of spinal anesthesia for transurethral prostatic resection. Eur J Anaesthesiol. 1992;9(1):7-13.

12. Munhall RS, Sukhani R, Winnie AP, et al. Incidence and etiology of failed spinal anesthetics in a university hospital: a prospective study. Anesth Analg. 1988;67(9):843-848.

13. Kavlock R, Ting PH. Local anesthetic resistance in a pregnant patient with lumbosacral plexopathy. BMC Anesthesiol. 2004;4(1): 1.

14. Fettes PD, Jansson JR, Wildsmith JA. Failed spinal anesthesia: Mechanisms, management, and prevention. $\mathrm{Br} J$ Anaesth. 2009;102(6):739-748
15. Hoppe J, Popham P. Complete failure of spinal anaesthesia in obstetrics Int J ObstetAnes. 2007;16(3):250-255

16. Trescot AM. Local anaesthetic "resistance". Pain Physician. 2003;6(3):291-293.

17. Berde C, Goldschneider K. Greco C, et al. Apparent Local Anesthetic Resistance in a Subgroup of Children and Adolescents with CRPS. A-1402, 2003

18. Trescot AM. Response to "Does scorpion bite lead to resistance to the effect of local anaesthetics?". Indian Journal of Anaesth . 2013;57(2):217.

19. Ragsdale DS, McPhee JC, Scheuer T, et al. Molecular determinants of state-dependent block of $\mathrm{Na}+$ channels by local anesthetics. Science. 1994;265(5179):1724-1728

20. Panditrao MM, Panditrao MM, Sunilkumar V, et al. Effect of previous scorpion bite(s) on the action of intrathecal bupivacaine: A case control study. Indian J Anaesth. 2013;57(3):236-240.

21. Bastawade DB, Jadhav SS, Sharma RM. "Scorpionida" (PDF) Zoological Survey in India. 2013;4(6):1-16.

22. Rodríguez-Ravelo R, Coronas FI, Zamudio FZ, et al. The Cuban scorpion Rhopalurus junceus (Scorpiones, Buthidae): component variations in venom samples collected in different geographical areas. $J$ Venom Anim Toxins Incl Trop Dis. 2013;19(1):13

23. Issin A. Local anaesthetic resistance in a young woman with history of scorpion bite. Indian J Anaesth. 2015;59(10):693-694.

24. Cestèle S, Gordon D. Depolarization differentially affects allosteric modulation by neurotoxins of scorpion alpha-toxin binding on voltagegated sodium channels. J Neurochem. 1998;70(3):1217-1226.

25. Cestèle S, Catterall WA. Molecular mechanisms of neurotoxin action on voltage-gated sodium channels. Biochimie. 2000;82(9-10):883-892. 